\title{
Towards Assessment of Open Educational Resources and Open Courseware based on a Socio-Constructivist Quality Model
}

\author{
Monica Vladoiu, Zoran Constantinescu \\ UPG University of Ploiesti, Romania \\ monica@unde.ro, zoran@upg-ploiesti.ro
}

\begin{abstract}
In this paper we introduce a rubric for assessing quality of open educational resources and open courseware based on our socio-constructivist quality model (QORE) that includes 70 criteria grouped in four categories related with content, instructional design, technology, and courseware evaluation. Quality is assessed from an educational point of view, i.e. how useful are such resources for various actors involved in educational processes taken into account their goals, objectives, abilities etc. QORE's focus is on the resources' potential to act as true open educational content available online that has a genuine educational value in this context. Several challenges of using this rubric for evaluation of such educational resources are discussed as well.
\end{abstract}

Keywords. open courseware, open educational resources, quality assurance, quality criteria, quality model, quality rubric

\section{$1 \quad$ Introduction}

Quality assurance of educational content is seen conventionally as being the responsibility of subject and instructional experts, but in the context of Open Educational Resources (OERs), OpenCourseWare (OCW), and Web 2.0, guaranteeing quality seems more and more a community endeavor based on the collaboration between experts in education, subject scholars, students, teachers, developers etc. both during and after the teaching and learning process through study groups and practice communities around the world [1]. Consequently, sense of community becomes more and more present in quality models of Web 2.0 applications and special focus has to be on user-centered, participatory nature of these emergent applications. However, such collaborative efforts are very difficult to undertake in absence of appropriate models, frameworks, and tools for evaluating quality of OERs and OCW, yet for the time being, no quality assurance framework that could provide support for various categories of users (learners, instructors, designers, faculty, evaluators etc.) is available.

In this paper we introduce a rubric for assessing OERs and OCW based on our socio-constructivist quality model, called QORE, which includes 70 criteria grouped in four categories related with content, instructional design, technology and courseware

adfa, p. 1, 2011.

(C) Springer-Verlag Berlin Heidelberg 2011 
evaluation. QORE had been introduced in [2], put to work in [3-7], and refined further for this work according with what we have learned from those use experiences. The paper is structured follows: the next section presents the related work, the third one introduces a detailed rubric for assessing quality of OERs and OCW that is based on our quality model, and the last one includes a discussion on the challenges of using this rubric, along with some conclusions and future work ideas.

\section{Related Work}

We overview here the related work and, because in terms of rubrics for OERs and OCW there are only very few similar works, we approach it in a larger sense of rubrics for learning objects and online courses as well. Thus, in $[8,9,10]$ the authors show that quality of learning objects may be improved by better educating their designers, by incorporative formative assessments and learning testing in design and development models, by taking into account the use context, and by providing summative reviews that should be maintained as metadata, which users can use when searching, sorting, and selecting learning resources. They have developed an instrument for reviewing quality of learning objects (called LORI) that incorporates several aspects as follows: content quality, learning goal alignment, feedback and adaptation, learners' motivation, presentation design, interaction usability, accessibility, reusability, and standards compliance. Furthermore, they use LORI within a suite of tools for collaborative evaluation that small evaluation teams (including subject matter experts, learners, instructional designers) use to produce an aggregated view of ratings and comments. Several works have tested LORI in particular cases $[9,11,12]$ and have pointed out that it can be used to reliably assess some aspects of learning objects and that using a collaborative assessment process can improve inter-rater reliability. In [13] seven categories for assessing OERs are provided, five of them being adapted from LORI (content quality, motivation, presentation design, usability, accessibility), while the other two are new: educational value and overall rating. Educational value refers to the resource's potential to provide learning, to its accuracy, clarity, and unbiasedness, while the overall evaluation captures the perceived usefulness of resources in educational contexts. Several other rubrics have been proposed for evaluating learning objects and/or online courses, neither of them being as visible in the literature as LORI [14-20]. Finally, Achieve, working together with the OER community, has developed a rubric, which provides for establishing both the degree of alignment to the Common Core State Standards and some quality aspects of OERs [21]. Recently, Achieve has jointed up with OER Commons to provide an online evaluation tool based on their rubric [22], and currently, each resource available in OER Commons may be assessed, the resulted evaluation is stored in a pool of metadata, and it may be shared through the Learning Registry with other interested repositories [23]. This rubric includes the next aspects: degree of alignment to standards, quality of explanation of the subject matter, utility of materials designed to support teaching, quality of assessment, quality of technological interactivity, quality of instructional and practice exercises, opportunities for deeper learning, and assurance of accessibility. 


\section{Quality Rubric for Evaluation of OERs and OCW}

We detail here the rubric based on the QORE quality model introduced in [2]. The QORE criteria may be used for quality assessment of either small learning units or entire courseware, and they are grouped in four categories related with content, instructional design, technology, and courseware evaluation. The majority of these criteria will be explained further on, along with their corresponding scoring scale. However, due to space limitations, some of the criteria will not be rubricated here. Nevertheless, they can be easily scored as it can be seen throughout this rubric. The not applicable N/A rating is used when a particular criterion cannot be evaluated for a specific resource. The fulfillment of each criterion is assessed on a scale between 0 and 5 , as follows: $0=$ absence, $1=$ inadequate, $2=$ satisfactory, $3=$ good, $4=$ very good and $5=$ excellent. Quality is assessed from an educational point of view, i.e. how useful are such resources for various actors involved in educational processes taken into account their goals, objectives, knowledge, abilities etc. QORE's focus is on the resources' potential to act as true open educational content available online that has a genuine educational value in this context. For the time being, the evaluation is subjective, being based, in our case, on many decades of evaluators' experience in Higher Education. However, this subjective evaluation seems to be the tendency of other works in this area $[8,13,21-23]$. First and foremost, there is necessary to point out that a good alignment between learning objectives, learning outcomes, teaching and learning activities, assessment methods, and curriculum standards (where appropriate) is crucial, and further on, when we use the term "align(ment)" we keep in mind all these aspects $[12,15,16,18,19]$.

Content Related (CR) criteria decide to what degree particular OERs/OCW allows learners to have engaging multiple learning experiences that provide content mastery.

CR1: Readability and understandability - the text is readable and it has clear writing, spelling and grammar are consistent and accurate, language is friendly and supportive, being easy to understand. The evaluation here is from an educational viewpoint.

N/A to non-textual resources;

$0=$ the text cannot be read due to various problems (for instance, font-related);

$1=$ the text can be read, but is almost impossible to understand (e.g. poor translation);

$2=$ the text is sufficiently readable and understandable;

$3=$ the text can be acceptably read and understood;

$4=$ the text can be read and understood properly;

$5=$ the text is excellently written, being highly readable and understandable.

Uniformity and appropriateness of language, terminology, and notations (CR2) is also important for most educational resources. The criteria $C R 3$ to $C R 8$ that are presented further on are not applicable (N/A) for resources that are small instructional units, being applicable only to courseware. 
CR3: Availability, easiness of locating and using the course syllabus, and its completeness is crucial, as various users may benefit from the information within it. $0=$ the syllabus is unavailable;

$1=$ the syllabus is available, but hard to locate and it contains deficient information;

$2=$ the syllabus is given and easy to locate, but it offers only a brief course overview;

$3=$ the syllabus is available, easy to locate, and contains almost complete information about the course (e.g. overview, learning objectives and outcomes);

$4=$ the syllabus is available, easy to locate and use, and contains complete information (prerequisites, overview, learning objectives and outcomes at course level, completion requirements, time advisory/learning unit); easy printable for convenience;

$5=$ in addition to 4 , the syllabus includes also learning objectives and outcomes at unit level, completion requirements, time advisory per learning unit, and, when applicable, course schedule, information on instructors, developers, evaluators etc., expectations of availability of and turnaround time for feedback.

The comprehensiveness of the course content (CR4) is also considered, i.e. whether the course content and assignments demonstrate sufficient wideness, deepness, and rigor to reach the educational standards, goals, objectives etc. being addressed. Modular course components (CR5) are desirable as they are units of content that may be distributed and accessed independently, giving each user the possibility to select easily the most suitable learning unit (CR6) and the opportunity to choose effortlessly the most appropriate learning path (CR7) that match her goals, needs, abilities, etc. and a variety of options to approach the resources: top-down, bottom-up or combined (CR8). Modularity may be approached at chapter level, i. e. each chapter is in a .pdf file, or at learning unit level, the latter being preferable.

CR9: Availability of proper assignments is important as well, as they are content items that enhance the main content. We measure here what the resource has to offer in terms of providing for engaging learning experiences that contribute to increased content mastery and learning efficiency (having assignments with solutions and builtin feedback being valuable in this respect). Based on the requirement for alignment, the focus is twofold: (1) establishing to what degree the assignments measure the achievement of the stated course learning objectives and outcomes, and (2) how appropriate they are in each particular case. For example, a composition is a proper assignment for evaluating writing skills, while a multiple choice quiz is suitable to test vocabulary knowledge [16].

N/A to resources that are not supposed to be assessed (for example, a guest lecture); $0=$ no assignments are available;

$1=$ an inadequate set of assignments that are weakly aligned and fitted is offered;

$2=a$ limited collection of sufficiently aligned and fitted assignments is offered;

$3=$ an average set of acceptably aligned and suitable assignments is provided;

$4=\mathrm{a}$ complete set of assignments both strongly aligned and suitable is available; $5=$ there is an excellent variety of fully aligned and highly appropriate assignments. 
When looking at a particular learning resource, other than courseware, which can be a small learning unit, a course module, a lesson etc. the quality model retains various features related to that small resource (CR10) that are of interest for users, such as accuracy $^{1}$, reasonableness ${ }^{2}$, self-containedness ${ }^{3}$, context $^{4}$, relevance ${ }^{5}$, multimedia inserts $^{6}$, interactive elements ${ }^{7}$, correlation with the entire course ${ }^{8}$, re-usability ${ }^{9}$, links to related relevant resources ${ }^{10}$ (audio, video etc.). To keep things simple we have these features placed on levels of significance: (1) accuracy, reasonableness, and relevance, (2) self-containedness, correlation with the course, (3) multimedia inserts, interactive elements, (4) context, re-usability, and (5) links to related relevant resources.

N/A to entire courseware;

$0=$ the resource is deficient in all the aspects relevant to the quality model;

$1=$ the resource is accurate, reasonable, and relevant;

$2=$ in addition to 1 , the resource is self-contained and correlated with the entire course;

$3=$ in addition to 2 , the resource contains multimedia inserts and interactive elements;

$4=$ in addition to 3 , information about proper context of use and re-usability is given;

$5=$ in addition to 4 , links to other related relevant resources are provided.

Instructional design (ID) related criteria address supporting learning theories, instructional design and strategies, and other pedagogical aspects of teaching and learning of that resource that provide for both increased educational value and high efficiency. Learning goal and objectives (ID1) outline the material and they should be aligned and suitably designed for the course level. They need to be: defined clearly both at course-level and unit level, consistent with each other, and explained from users' perspective. They help users focus their efforts and clarify expected quantifiable learning outcomes, and, hence instructions on how to achieve them must be stated clearly [14, 16, 18, 20]. Learning outcomes (ID2) state the learner's achievements after performing a learning activity, i. e. what learners will know and/or will be able to do as a result of such an activity, in terms of knowledge, abilities, skills, attitudes, and values. The course learning objectives describe outcomes that are measurable/observable/demonstrable and that are clearly stated and explained to be easily understood by students [14, 18, 19]. Moreover, learning outcomes must be aligned and relevant, i.e. useful and appropriate for the intended users, and, of course, achievable, realistic, and appropriate to the rigor and the breadth of the learning objective. Learning outcomes must include, if necessary, the skills required (1) for using the resource, in a manner that is consistent with a stated pedagogical paradigm, (2) for proving achievement of the learning objectives, and (3) for linking new knowledge with existing knowledge and future contexts (element of reflection). Pedagogically, covering a good range of learning outcomes in both Bloom's taxonomy and Fink's taxonomy is welcome [19].

\section{ID1: Learning goal and objectives}

N/A to resources that have no explicit learning goal and objectives;

$0=$ no statement about learning goal and objectives;

$1=$ the learning goal and objectives are stated only at course level;

$2=$ in addition to 1 , they are stated, partially, at unit level, being sufficiently aligned; 
$3=$ in addition to 2 , they are almost completely aligned, and match the degree's level; 4=in addition to 3, they are consistent, stated at both levels, and aligned completely; $5=$ in addition to 4 , users are provided with explanations and how-to-reach roadmap.

\section{ID2: Learning outcomes}

N/A to resources that have no explicit learning outcomes;

$0=$ no statement about the learning outcomes is available;

$1=$ the learning outcomes are stated only at course level;

$2=$ the learning outcomes are stated and explained clearly at course level and, partly, at unit level, being partially quantifiable and, to some extent, achievable, realistic, and appropriate; they are sufficiently aligned and relevant;

$3=$ the learning outcomes are stated and explained clearly at course level and, partially, at unit level, being suitably quantifiable and, to some extent, achievable, realistic, and appropriate; they are almost completely aligned and have acceptable relevance;

$4=$ the learning outcomes are stated and explained clearly both at course level and at unit level, being completely quantifiable and fully achievable/realistic/appropriate; they are completely aligned and have strong relevance; they cover a proper range in both Bloom's taxonomy and Fink's taxonomy;

$5=$ in addition to 4, they cover most of the outcomes in both Bloom's taxonomy and Fink's taxonomy; moreover, they include extra-skills (e.g. reflection).

The educational resources are ought to provide for multiple opportunities for learners to be actively engaged in efficient learning processes, having meaningful and authentic learning experiences, which address multiple learning styles, during which they undertake various appropriate instructional activities (ID3) such as problem- or project-based learning, e-simulations, webcasts, scavenger hunts, guided analysis, guided research, discovery learning, collaborative learning groups, case studies, serious games, portfolios etc. Other users may benefit also: instructors, developers etc. There is likely that these activities promote the achievement of the stated learning objectives and outcomes (with which they are aligned) [14, 16, 17, 19]. As shown before, a good alignment between learning objectives, learning outcomes, teaching and learning activities, and assessment methods is necessary. Consequently, learning objectives must have corresponding appropriate assessments [18, 19]. Ongoing multiple assessment strategies are desirable to measure content knowledge, skills, abilities, attitudes, and values. Students' self-assessments (similar to the final ones) and peer feedback opportunities throughout the course are needed. When possible, options among assignments should be provided to allow learners with different interests, backgrounds, and personal learning styles to demonstrate their proficiency [18]. Learners learn more effectively if they receive frequent, meaningful, and rapid feedback, which may come from the instructor directly (when appropriate) or from assignments and assessments that have built-in feedback [16]. Hence, appropriate assessments and self-assessments means, with or without solutions (ID4) are highly desirable, as a quality feature, when aiming at mastery of educational content. Users may be also interested in the supporting learning theory (behaviorist, cognitivist, constructivist, humanist and motivational etc.) and in the instructional design model 
(ADDIE, ARCS, ASSURE etc.) that have been used to develop that particular educational resource (ID5). Effective instructional strategies (direct, indirect or interactive instruction, independent study, experiential learning etc.) may also impact on the efficiency of instruction (ID6). Moreover, learning experiences that provide for reflective and deeper learning (ID7) will always add to the overall quality of educational resources. Under the reflection perspective, the desired outcome of education becomes the construction of coherent functional knowledge structures adaptable to further lifelong learning $[2,5]$. Deeper learning is expected to prepare learners for mastering of core content, for thinking critically and solving complex problems, for working collaboratively, for communicating effectively, for learning how to learn, and for developing academic mindsets [24].

Technology Related (TR). Both OERs and OCW are expected to benefit from ICT technologies, to have user-friendly interfaces, to comply with standards for interoperability (TR1), and to provide for appropriate access for all learners (accessibility TR2). Extensibility (TR3) of each educational resource, aiming at expanding learning opportunities, from a technological point of view, refers to easiness of adding content, activities, and assessments for designers, developers, teachers, learners etc. A high quality user interface is based on technical aspects related to the capabilities of the supporting hardware, software, and networking (TR4: user interface's basic technological aspects). A clear specification of the supporting technology requirements at user's end (both hardware and software) - TR5, along with the prerequisite skills to use the supporting technology (TR6) are useful to help users understand how the resource should be used to benefit fully from its content. High quality OERs and OCW are expected to work smoothly on a variety of platforms (multi-platform capability TR7). Having a true engaged learning relies on multiple learners' opportunities to interact with the content and with other learners, which is not possible without a suite of rich supporting tools that provide for adaptation and personalization (TR8).

CourseWare evaluation (CW). Despite of the original claim of just offering high quality educational materials, all major OER/OCW initiatives have recently become more involved with their learners. Hence, regular assessment of effectiveness of open courseware becomes essential, along with using the results for further improvements.

CW1:Courseware overview: content scope $e^{1}$ and sequence ${ }^{2}$, intended audience ${ }^{3}$, grade level $^{4}$, periodicity ${ }^{5}$ of content updating, author's credentials ${ }^{6}$, source credibility, multiple-languages ${ }^{8}$, instructor facilitation ${ }^{9}$ or semi-automated support ${ }^{10}$, suitableness for self-study ${ }^{11}$, classroom-based ${ }^{12}$ study, and/or peer collaborative ${ }^{13}$ study, time requirements $^{14}$, grading policy ${ }^{15}$, instructions on using ${ }^{16}$ the courseware, reliability ${ }^{17}$, links to other ${ }^{18}$ educational resources (readings, OCW, OERs etc.), alignment ${ }^{19}$

N/A to small learning units;

$0=$ the courseware is deficient in all its aspects relevant to the quality model;

$1=$ basic information about the content scope $^{1}$ and sequence ${ }^{2}$, intended audience ${ }^{3}$, and grade level ${ }^{4}$ is available; 
$2=$ in addition to 1 , information about the author's credentials ${ }^{6}$, source credibility ${ }^{7}$, and alignment ${ }^{19}$ is provided;

$3=$ in addition to 2 , information about the instructional paradigm ${ }^{9-13}$ is offered;

$4=$ in addition to 3 , information on time advisory ${ }^{14}$, grading $^{15}$, how-to use the courseware $^{16}$, and reliability ${ }^{17}$ is given;

$5=$ in addition to 4 , information on updating periodicity ${ }^{5}$, multiple-languages ${ }^{8}$ and links to other related educational resources ${ }^{18}$ is offered.

Availability of prerequisite knowledge (CW2) and of required competencies (CW3) is useful for users at the beginning of a learning process. Matching the course schedule, if any, with learner's own pace (CW4), may be also needed in some contexts. Another useful criterion regards the terms of use (service) $-C W 5$, i.e. availability of repository or institutional policies with respect to copyright and licensing issues, security for primary, secondary and indirect users, anonymity, updating and deleting personally identifiable information, age restrictions, netiquette, etc. OERs and OCW that show freeness of bias and advertising and cultural sensitiveness (CW6) are also desirable. Suitable design and presentation of educational content ( $C W 7)$ is also wanted, along with user interface richness (style) - CW8 - as it is defined by its navigational consistency $^{1}$, friendliness ${ }^{2}$, multimedia inserts ${ }^{3}$, interactivity ${ }^{4}$, and adaptability ${ }^{5}$ (both to user's needs and context). Another quality criterion is concerned with the option to provide, or aiming to provide, a formal degree or a certificate of completion (CW9). Participatory culture and Web 2.0 facets $(C W 10)$ are also important and they can be detailed as it can be seen beneath.

CW10: Participatory culture and Web 2.0 facets: contribution to the content ${ }^{1}$, collection of users' feedback, ${ }^{2}$, collaboration with fellows ${ }^{3}$, sharing the development ${ }^{4} /$ sing $^{5}$ experience

N/A to resources that do not comply with Web 2.0 paradigm;

$0=$ the courseware do not show any of the important Web 2.0 features;

$1=$ user feedback is collected to be used for further improvements;

$2=$ in addition to 1 , (some) collaboration with fellows is possible;

$3=$ in addition to 2 , (some) contribution to the content from developers is allowed;

$4=$ in addition to 3 , contribution from learners is feasible;

$5=$ in addition to 4 , sharing using and development experience is possible.

\section{Conclusions, Challenges, and Future Work}

Helping lifelong learners to find efficiently and effectively the most relevant OERs and/or OCW according with their goals, needs, knowledge, abilities, etc. is crucial, and communities play a central role in this process, by collaborating distributedly within virtual environments and by socially constructing and sharing knowledge related to this kind of educational content. We introduced here a rubric based on our socio-constructivist quality model for quality assessment of OERs and OCW, as one of our first steps towards the construction of a quality evaluation framework, which 
may help various users to utilize, modify, re-use, re-mix, design, evaluate, compare, recommend etc. OERs and $\mathrm{OCW}$, while pursuing their educational goals. We are currently focusing our efforts on developing a working prototype of this framework, which aims at classifying and recommending OERs and OCW based on both quality evaluations performed by various users (instructors, learners, instructional experts, designers, developers etc.) using this rubric and collaborative feedback from peers $[5,7]$. One huge challenge in this moment is the "contributing problem", i. e. how to have as many reviewers as possible performing quality assessment using our rubric. Thorough quality assessment of educational resources takes time, effort, and expertise, and therefore such quality reviews are not very common, especially when a high number of criteria is considered. To cope with this shortcoming we intend to develop a rubric-applying tool that facilitates human assessment so that potential evaluators, hopefully, will be keener on performing evaluations. This tool could provide them with samples of various similar pieces of educational content that pertain to different quality classes, along with their score and a brief explanation of that score. We also consider automatic evaluation of some of the criteria, which can be achieved by parsing intelligently each resource's website. Human evaluators may keep the results of these automatic assessments or they may change them to reflect their viewpoint. This could help also with incomplete evaluations that have scores only for some of the quality criteria. To obtain assessments from learners' point of view we think to involve, at first, our undergraduate and graduate Computer Science students and to have them assessing OERs and OCW for their semester projects. The first base is having gathered in a common pool of resources and evaluated several OCW and OERs (around 10 resources per subject) that are necessary to graduate majoring in Computer Science. Further on, we consider automating some activities of our framework: the federated retrieval of OERs/OCW based on a retrieving taxonomy, capturing context and knowledge about user, and so on. Another direction to work on is concerned with objective measurements that could be included in the quality model: number of accesses, time spent with a resource, number of bookmarks, number of times a bookmark is followed, number of citations etc. Nevertheless, the semantics of such information has to be modeled properly if it is ought to complement seamlessly the explicit quality ratings. A weighting mechanism between the assessments of various users could be also useful to favor, for instance, a subject-matter expert's or an instructional designer's evaluation when compared with one of an anonymous on-line user. False positive (unfair) evaluations need to be banned somehow and we think about using Bayesian Belief Networks for that. Another idea we would like to pursue refers to refining our quality model towards a hierarchical approach, aiming at categorizing open educational resources for specific contextual goals and needs. The prototype will be used both in formal and informal environments, then evaluated, and, hopefully, the viability of our approach will be validated.

\section{References}

1. Piedra, N., Chicaiza, J., Tovar, E., Martinez, O.: Open Educational Practices and Resources Based on Social Software: UTPL Experience, $9^{\text {th }}$ ICALT 2009, pp.497-498 (2009) 
2. Vladoiu, M.: Quality Criteria for Open Courseware and Open Educational Resources. In $11^{\text {th }}$ ICWL 2012 Workshops, to appear in LNCS 7697, Springer, Heidelberg (2013)

3. Vladoiu, M., Constantinescu, Z.: Evaluation and Comparison of Three Open Courseware Based on Quality Criteria. In Grossniklaus, M., Wimmer, M. (eds.), 12 $2^{\text {th }}$ ICWE 2012 Workshops - QWE 2012, LNCS 7703, pp. 204-215, Springer, Heidelberg (2012)

4. Vladoiu, M.: Towards Assessing Quality of Open Courseware. In 11 ${ }^{\text {th }}$ ICWL 2012 Workshops, to appear in LNCS 7697, Springer, Heidelberg (2013)

5. Moise G., Vladoiu M., Constantinescu Z.: Multi-Agent System for Evaluation and Classification of OERs and OCW Based on Quality Criteria, submitted for publication (2013)

6. Constantinescu, Z., Vladoiu M..: Evaluation and Comparison of Eight Open Courseware based on a Quality Model. In $9^{\text {th }}$ Int'l Scientific Conference eLearning and Software for Education - ELSE 2013, Ed. Universitara, Bucharest (2013)

7. Vladoiu M., Constantinescu Z., Moise G.: QORECT - a Case-Based Framework for Quality-based Recommending OCW and OERs, C. Bădică, N. T. Nguyen, and M. Brezovan (eds.): ICCCI 2013, LNAI 8083, pp. 681--690. Springer, Heidelberg (2013)

8. Nesbit, J.C., Li, J.Z. and Leacock, T.L.: Web-Based Tools for Collaborative Evaluation of Learning Resources, Journal of Systemics, Cybernetics and Informatics, 3(5) (2005), http://www.iiisci.org/journal/sci/Contents.asp?var=£previous=ISS2829

9. Vargo, J., Nesbit, J. C., Belfer, K., Archambault, A.: Learning Object Evaluation: Computer-Mediated Collaboration and Inter-Rater Reliability. International Journal of Computers and Applications, 25(3), 1-8 (2003)

10. Leacock, T. L., Nesbit, J. C.: A Framework for Evaluating the Quality of Multimedia Learning Resources. Educational Technology \& Society, 10 (2), 44-59 (2007)

11. Akpinar, Y.: Validation of a Learning Object Review Instrument: Relationship between Ratings of Learning Objects and Actual Learning Outcomes. Interdisciplinary Journal of E-Learning and Learning Objects, 4(1), 291-302 (2008).

12. Krauss, F., Ally, M.: A Study of the Design and Evaluation of a Learning Object and Implications for Content Development. Interdisciplinary Journal of E-Learning and Learning Objects, 1(1), 1-22, (2005)

13. Burgos Aguilar, J. V.: Rubrics to evaluate OERs (2011), www.temoa.info/sites/default/files/OER_Rubrics_0.pdf

14. QM Programs, Quality Matters Rubric Standards 2011-2013 edition, http: / /www . qmprogram.org/rubric

15. Buzzetto-More, N.A. \& Pinhey, K. (2006). Guidelines and standards for the development of fully online learning objects. Interdisciplinary Journal of Knowledge and Learning Objects, 2, 95-104, (2006) http://ijklo.org/Volume2/v2p095-104Buzzetto.pdf

16. Minnesota Assessment Center, Peer Course Review Rubric - Standards with Point Value and Annotations, eassessment.project.mnscu.edu

17. CSU Faculty Development Center, Quality Online Learning and Teaching (QOLT) Rubric Summary, fdc.fullerton.edu/teaching

18. CSU-Chico, Instruction Design Tips for Online Learning and Rubric for online instruction, http://www.csuchico.edu/roi/

19. University of Utah, TLT Grant Hybrid Course Proposal Rubrics, https://eq. utah .edu

20. Central Piedmont Community College, CPCC eLearning: Quality Course Review (QCR), www.cpcc.edu/elearningcommunity/quality/QCR_Rubric.pdf

21. ACHIEVE, http://www.achieve.org

22. OER Commons, http://www.oercommons.org

23. Learning Registry, http://www.learningregistry.org

24. The Hewlett Foundation, Deeper learning competencies (2013), www . hewlett.org 